\title{
An Empirical Model of Morphologies of Planetary Nebulae
}

\author{
C.Y. Zhang and S. Kwok
}

University of Calgary, Calgary, Canada

The variety of different morphologies of planetary nebulae (PNs) have always fascinated researchers in the field. Recent progress in CCD imaging techniques has resulted in a large number of homogeneous optical images of more than a few hundreds PNs (Balick 1987, Schwarz, Corradi and Melnick 1992). In addition, two major radio surveys using the Very Large Array (VLA) by Aaquist and Kwok $(1990,1991)$ and by Zijstra, Pottasch and Bignell (1989) have revealed detailed morphologies in the radio, including many compact star-like PNs. This treasure of data sets contains useful information about morphologies, density distributions, formation and evolution of PNs, yet to be fully explored.

While approaches using purely descriptive terms, such as, round, elliptical, bipolar, and irregular, are commonly taken to classify PNs into different categories, a more quantitative approach will be more useful in furthering our understanding of the PN phenomenon.

In this paper we have applied an empirical model of the Prolate Ellipsoidal Shell (PES) to match the morphologies of $110 \mathrm{PNs}$, ranging from round, elliptical to bipolar ones, in the optical $\mathrm{H}_{\alpha}$ and radio continuum emission. There are six main parameters in the model, that is, the ratio of equator-to-polar axis of the inner cavity, $\epsilon$, the relative shell thickness along the minor axis, $\Delta$, the parameter, $\alpha$, controlling the steepness of the angular density gradient, the ratio of the polar-to-equator axis density, $\beta$, the power index of the radial density law, $\gamma$, and the angle between the polar axis and the line of sight, $i$. In addition, one can adjust two more parameters, $\delta_{\theta}$ and $\delta_{\phi}$ to control the degree of ellipsoidal asymmetry along the polar and equator directions. For definitions of these parameters and details of the model, see Appendix of Aaquist and Kwok (1996).

It is found from the model fitting that the variety of vastly different morphologies can be explained by the combination of projection effect and the different polar-to-equator density contrasts within this single, unified PES model. The results of the model fitting to more than one hundred PNs have allowed a statistical study of PN morphologies, based on quantitative classifications. In particular, the Kolmogolov-Smirov test shows that Type I and non-Type I PNs are different in that Type I objects tend to have larger $\alpha$ (steeper angular density gradient) and smaller $\beta$ (greater equator-to-polar density contrast), compared to non-Type I objects.

\section{REFERENCES}

Aaquist, O. B. and Kwok, S. 1990, A\&AS, 84, 229.

Aaquist, O. B. and Kwok, S. 1996, ApJ, 462, 813.

Balick, B., 1987, AJ, 94, 671.

Zijlstra, A. A., Pottasch, S. R. \& Bignell, R. C. 1989, A\&AS, 79, 329. 